\title{
LA BONNE FOI DANS LA FORMATION DU CONTRAT EN DROIT BRÉSILIEN
}

\author{
Antonio Junqueira de Azevedo \\ Professeur Titulaire du Département du Droit Civil \\ de la Faculté de Droit de l'Université de São Paulo \\ Directeur de la Faculté de Droit de l'Université de São Paulo
}

\section{L'utilisation de la notion de bonne foi dans le droit brésilien}

1.1. Dans le droit brésilien, "la notion de bonne foi dans les rélations entre des particuliers est un principe juridique, mais il n'est pas formulé comme règle d'aplication générale.

Dans le Code Civil brésilien, dont la vigueur est initié depuis le ler. janvier 1917, élaboré sous l'influence des idées pandectistes à la deuxième moitié du siècle passé, il n'y a pas de règle générique référant expressement la bonne foi dans la formation ou dans l'éxécution des contrats comme celles des articles ou paragraphes 1.134 du Code Civil français, 242 du B.G.B., $1.337 \mathrm{du}$ Code Civil italien et 227, du Code Civil portugais.

Il y a, dans cette omission du Code Civil brésilien, un reflet de la mentalité capitaliste de la deuxième moitié du siècle XIX, plus préoccupée avec l'assurance de la circulation et dévéloppement des rélations juridiques qu'avec la justice matérielle des cas concrets, car, à la vérité, le Code Commercial brésilien, beaucoup plus ancien que le Code Civil (le Code Commercial est de 1850), avait déjà une règle générique sur la bonne foi c'est l'article 131, sur l'interprétation contractuelle.

Quand il traite des sujets spécifiques, comme contrat d'assurance (arts. 1.443 à 1.446), contrat de societé (art. 1.404), acquisitions " $a$ non domino" (art. 622), paiement de l'indu (art. 968), possession (arts. 490 et suivants), prescription acquisitive (arts. 500 et suivants), dette de jeu (art. 1.477) et coetera, le Code Civil lui même prévoit la bonne foi pour certaines conséquences juridiques. Il s'agit, pourtant, dans tous ces cas, sauf les deux premiers (contrat d'assurance et contrat de société), de la dite bonne foi subjective, c'est-à-dire, de cet état psycologique relatif à la connaissance ou à la non connaissance et à l'intention ou au manque d'intention de quelqu'un. 
Nous devons dire encore que, actuellement, comme conséquence du changement d'esprit, la récente loi de protéction au consommateur - qui, à cause de son amplitude, est nommée Code de Protéction au Consommateur (Loi n. 8.078 de 1990), a le principe de la bonne foi refléti dans de nombreux articles. Ici il s'agit, au fait, de la bonne foi objective, c'est-àdire la bonne foi comme règle de conduite.

1.2. Spécifiquement, lors des pourparlers et négociations conduisant à la conclusion du contrat, il est important de souligner dès maintenant mais pour dévéloppement postérieur - que, dans le droit brésilien, il y a une règle expresse selon laquelle l'offre de contrat oblige le promettant. Toutefois, hors de la question de l'offre et, evidemment, d'éventuelles conventions expresses par les parties sur la négociation, les tribunaux brésiliens ne recconaissent aucun lien d'obligation entre les pré-contratants. Donc, la rupture des négociations et le repentir avant la conclusion du contrat sont libres.

La rélation entre les pré-contratants aura seulement son éfficacité juridique du côté négatif ou pathologique, c'est-à-dire, seulement par le côté du devoir de ne pas se comporter d'une manière socialement condamnable. Ce devoir n'est pas une obligation proprement dite ("vinculum iuris"). Il est sanctioné par l'action d'indemnisation (action "ex delicto" et "ex quasi delicto") de l'article 159 du Code Civil. Tant aux pourparlers comme au moment même de la conclusion du contrat, la situation au Brésil est vue du côté de la mauvaise foi, soit: l'entendement est celui selon lequelle seulement est responsable pour les dommages-intérêts celui qui a agit de mauvaise foi dans la phase de la formation contractuelle (en verité, s'agit, ici encore, d'un aspect de la bonne foi subjective).

Cette intelligence est extraordinairement facilitée par la règle de l'article 159 du Code Civil sur l'acte illicite qui est d'ampleur peu commune dans les législations d'autres pays. Cette règle, également connue par tous les juristes et par les simples étudiants de droit au Brésil, préscrit que tous ceux qui, par acte ou omission volontaires, par néglicence ou imprudence, causent des préjudices ou violent le droit, sont obligés à réparer le dommage. Elle est, dans le champs du droit privé, l'expression légale du principe général de droit du respect au prochain et à ses biens (du "alterum non laedere", deuxième principe général de droit selon Ulpianus et les stoiciens). L'indemnité sera totale 
et, naturellement, étant donné que la responsabilité est aquilienne, la preuve de la faute est à la charge de l'auteur de l'action et la prescription est celle des actes illicites.

\section{Des efetts de la manque de bonne foi dans la formation du contrat}

2.1 . Lorsque le contrat n'est pas conclu, par la suite de la rupture des pourparlers ou des négociations préliminaires, on peut, pourtant, obtenir indemnité pour acte illicite, allégant une conduite déloyale ou abusive. Très souvent on fait donc référence à la théorie de Von Jhering sur la faute "in contrahendo". Hors de l'hypothèse de l'acte illicite en laissant de côté la question de l'offre et d'éventuelles conventions expresses entre les parties sur les négociations - la rupture unilatérale et le repentir sont absolument libres. Si la rupture advient avec une juste cause, sans faute, il n'y a pas de responsabilité pour les dommages causés à l'autre partie; le droit brésilien n'a pas la règle générale de bonne foi, une "notion ouverte" destineé beaucoup plus au juge qu'aux parties.

Si le contrat est conclu et s'il est annulable par vice, le légitimé pour les actions par exemple, celui qui est trompé par dol principal ou demande l'annulation ou ne la demande pas. S'il ne la demande pas, il n'y a pas de conséquences, soit, si l'intéressé a choisi le maintien du contrat, il ne peut pas, à la fois, obtenir n'importe quelle indemnité pour dommage causé pour la mauvaise foi de la partie contraire. S'il la demande, la sentence constitutive négative a l'efficacité rétroactive, ex tunc; il y a la restituition des parties à l'état qu'elles avaient avant et, ceci n'étant pas possible, il y a l'indemnité de l'équivalent (article 158 du Code Civil). En plus du retour au statu quo ante, le légitimé peut, cumulativement, en se basant à l'article 159 du C.C., demander dommages-intérêts résultants de la responsabilité délictuelle de tout ce qu'il n'a pas récuperé avec l'annulation.

Ce qui est le plus intéressant c'est que, quand le dol ou la coaction ne sont pas déterminants de l'affaire - et donc se réfèrent à un point sécondaire l'affaire n'est pas annulable, mais celui qui est prejudiqué, quand même, peut demander dommages-intérêts à cause du délit de l'auteur de la lésion. 
3. Contenu concret de la nommée obligation de bonne foi dans la phase de formation contractuelle

3.1.

Le principe de la bonne foi dans la formation contractuelle peut se dévélopper en règles spécifiques: sur le devoir d'informer la partie adverse, sur le devoir de mantenir le secret des conversations, sur le devoir de faire savoir le changement d'avis sur l'affaire, et coetera.

Une règle sur le devoir d'informer sur fait ou qualité essentiels existe dans le droit brésilien "a contrario sensu" de l'article 94 du C.C., qui détérmine que l'omission par dol soit cause d'annulation. L'article mentioné préscrit: "Dans les actes bilatéraux, 'le silence' voulu d'une partie sur fait ou qualité que l'autre partie ignore est omission dolosive, si on prouve que sans elle le contrat "l'aurait pas été célébré".

Le devoir d'informer existe aussi, expressement, dans le contrat d'assurance (arts. 1.443 à 1.446 du C.C.). Cette règle existe, pourtant, depuis 1917, mais nous observons que, dans ce cas, comme dans le cas de l'offre, l'existence de la règle spécifique rend inutile l'appel à la règle générique de la bonne foi.

Actuellement, dans le Code de Protection au Consommateur, il y a de nombreuses règles imposant le devoir d'information; de cette forme, l'art. 6, entre les droits fondamentaux du consommateur, inclut le droit "à l'information convenable et claire sur les différents produits et services avec spécification juste de quantité, caracteristiques, composition, qualité et prix, et aussi des risques qu'ils présentent". Il y a encore tout un chapitre (Chapitre V) Des Pratiques Commerciales, avec l'exigence de véracité dans l'offre (Séction II) et dans la publicité (Séction III).

3.2.

D'un autre côté, une règle sur le devoir de confidentialité à l'égard des informations obtenues de l'autre partie n'existe pas, malheureusement, dans la législation brésilienne. Ce devoir, sauf dans les cas naturels du secret professionnel, n'est ni même caractérisé dans la jurisprudence et dans la doctrine. Les cas concrets auront leur solution dans le fondement légal de l'article 159 du C.C. et du conséquent devoir général de n'avoir pas mauvaise foi. 
3.3.

D'autres règles spécifiques sur la manière d'agir au cours des négociations et la conclusion du contrat n'existent pas. Le Code de Protection au Consommateur, établit pourtant quelles sont les "pratiques abusives" (articles 39-41), entre lesquelles, la suivante: "il est défendu au fournisseur de produits ou services de se prévaloir de la faiblesse ou de l'ignorance du consommateur, par égard à son âge, santé, préparation intelectuelle et condition sociale, pour lui vendre ses produits ou services".

La distinction entre obligation de moyen et de résultat, bien que connue, n'est pas adoptée dans la législation brésilienne, n'apportant donc, aucune différence à ce qui a été dit jusqu'ici.

\section{Sanctions}

4.1. La sanction, dans le droit brésilien, vise remettre le préjudiqué à l'exacte situation qu'il avait avant le fait d'où s'origine le préjudice. Il en résulte que, si le contrat n'a pas été conclu et s'il y a possibilité de caractériser la rupture des négociations comme acte illicite (art. 159 du C.C.), les dommages-intérêts incluront tout ce que la partie lésée a perdu (damnum emergens) et tout ce que raisonnablement elle n'a pas gagné (lucrum cessans). "Ce que raisonnablement elle n'a pas gagné" n'est pas, naturellement, ce qu'elle obtiendrait avec le contrat mais ce qu'elle obtiendrait si les négociations n'eussent pas été réalisées. Par ailleurs, dans le droit brésilien, on n'adopte ni l'expression, ni le concept d'intérêt négatif du droit allemand.

4.2.

Si le contrat est conclu et, par la suite, annulé en raison d'un vice de formation, il faut que l'on rende tout ce qu'on a reçu et, si cela n'est pas possible, l'équivalent. D'ailleurs, s'il y a des préjudices inconverts pour le simple retour au statu quo ante, c'est bien possible, étant caractérisé l'acte illicite, de demander ce qui manque avec fondement à l'article 159 du C.C.

4.3.

Si le contrat est conclu et maintenu, car le vice n'est pas essentiel (et donc, le contrat n'est pas annulable), comme c'est déjà dit (n. 2.3.), on peut demander dommages-intérêts à cause des mauvaises conditions dans lesquelles l'affaire a été faite (voir l'article 93 du C.C. pour le dol accidentel). 
4.4. Observons que, dans le droit brésilien, le dol de tiers, inconnu de la partie à qu'il fait du profit, ne conduit pas à l'annulation mais le préjudiqué a l'action de dommages-intérêts contre le tiers par l'article 159 du C.C.. La coaction pratiquée, par tiers, différemment, néanmoins soit elle inconnue de la partie à qui elle fait profit, conduit à l'annulation mais, pour lors, c'est celui qui voit le contrat annulé qui a l'action de dommages-intérêts contre le tiers.

4.5 .

Dans un cas, la sanction arrive à être étonnante: si les deux parties ont agi par dol, la sanction est... la validité du contrat! (article 97 du C.C.: "Si toutes les deux parties ont agi par dol, aucune des deux ne peut pas l'alleguer pour annuler l'acte ou demander l'indemnité").

4.6.

N'importe queile hypothèse d'indemnité, le quantum est fixé objectivement selon la somme des préjudices ou selon la clause de pré-fixation de dommages-intérêts. On ne peut pas augmenter ou diminuer le quantum selon le dol ou degré de faute de l'agent. Dans le cas échéant, le juge demande l'arbitrage des experts.

\section{La question de l'offre}

Au contraire de l'ordre juridique d'autres pays, le brésilien n'a pas besoin de recourir au principe de bonne foi pour solutionner le problème de la retraite de l'offre avant l'acceptation. L'article 1.080 du C.C. détermine: "L'offre de contracter oblige le proposant, si le contraire ne résulte pas de ses termes, de la nature de l'affaire ou des circonstances du cas".

Quoiqu'il y ait, au champs de la preuve, évidemment, quelques difficultés pour caractériser l'offre attachante, on peut dire, techniquement, que le proposant par acte unilatéral crée, dans le patrimoine du proposé, un droit expectatif ou potestatif de conclure le contrat. C'est spécialement clair dans les cas d'offre irrévogable ou avec un délai déterminé d'éfficacité. Il suit que la retraire de l'offre entraine la responsabilité obligationelle, c'est-à-dire, non aquilienne ou extracontractuelle. 
Observons, pourtant, que l'obligation qui surgit de l'offre n'a pas l'effet propre d'attacher le proposant au futur contrat si la retrait de l'offre se donne avant l'acceptation. L'obligation devient dommages-intérêts.

Différemment, le Code de Protection au Consommateur donne éfficacité pleine à l'offre. Selon son article 35, et si le consommateur le veut, le fournisseur restera obligé à l'accomplissement forcé dans les termes de l'offre (et d'ailleurs, la publicité, ou la présentation pour la consommation, de biens ou services, s'équiparent à l'offre).

\section{Le pré-contract}

Dans le droit brésilien, les négociations préliminaires ne se confondent aucunement avec le précontrat. Celles là, comme c'est déjà dit, sont traitées sous l'optique de la nommée responsabilité extracontratuelle de l'article 159 du C.C., pendant que le précontrat est déjà un contrat; il crée attachement juridique obligation entre les parties avant même de n'importe quel manque d'accomplissement.

Le précontrat est très commun au Brésil. En 99,9\% des cas de ventes d'immeubles, avant le contrat définitif, ce fait un précontrat, dit compromis ou promesse d'achat-et-vente, où l'on prévoit le payement du prix en parcelles (et l'acte notarié d'achat-et-vente ne se fait qu'après le payement intégral du prix).

Tous les précontrats ont une éxécution spécifique (selon l'art. 639 du Code de Procédure Civile, la sentence aura le même effet du contrat qui devrait être signé).

Il y en a qui ont, même, une action propre, par exemple, l'adjudication compulsoire pour les promesses d'achat-et-vente.

\section{La bonne foi et les Tribunaux Supérieurs}

Le Suprême Tribunal Fédéral a actuellement, au Brésil (Constitution de la République Fédérative du Brésil, qui est de 1988), compétence spécialement tournée vers le contrôle constitutionnel des lois, actes et décișions; ce n'est pas, donc, un tribunal similaire à la Cour de Cassation 
Française. Du reste, la constitutionnalité de nos lois n'est pas examinée par avance hors du Pouvoir Législatif.

Au dessous du Suprême Tribunal Fédéral, il y a le Supérieur Tribunal de Justice qui, par le moyen des "recours spéciaux", examine des questions de droit et n'examine proprement pas les questions de fait, dans les procès déjà décidés par les tribunaux inférieurs. Il s'en suit de l'exposé que, étant donné que la bonne foi est normalement une question de fait, difficilement elle est examinée dans les tribunaux supérieurs. Néanmoins, cet examen peut arriver dès que, avec l'emploi de beaucoup d'argutie, l'on réussisse à démontrer que, par avance, il y a un problème de qualification juridique dans la matière décidée pour l'instance inférieure.

\section{Autonomie privée et principe de la bonne foi}

La doctrine et la jurisprudence brésiliennes ne se sont pas dédiées à l'importante question de savoir quels seraient les limites de l'autonomie privée dans l'élimination ou l'atténuation des conséquences du principe de la bonne foi.

Il nous semble, premièrement, en essayant d'interpréter "l'esprit du droit brésilien" une activité dangereuse que toutes les conventions ou clauses qui visent directement éliminer, ou tout simplement diminuer l'exigeance de bonne foi dans la formation et éxécution des contrats sont nulles.

Antérieurement au Code Civil, on disait déjà qu'étaient nulles toutes les conditions contraires aux bonnes coutumes et à l'ordre public; les notions de bonnes coutumes et d'ordre public, malgré leurs imprécisions, intègrent l'ordre juridique brésilien (article 17 de la Loi d'Introduction au Code Civil); les actes contraires aux bonnes coutumes et à l'ordre public sont illicites (= contraires à la loi), aussi bien que les conventions ou clauses qui visent éliminer ou diminuer la bonne foi.

Par ailleurs, les conventions ou clauses de ne pas indemniser et les conventions ou clauses qui limitent l'indemnité (par exemple, la clause de pré-fixation de dommages-intérêts) sont valides, car elles ne constituent pas, en principe, aucune autorisation pour agir illicitement. L'accord est valide sauf certaines hypothèses spécifiques (par exemple, dans les contrats de transport, les 
clauses de ne pas indemniser sont nulles court abrégé n. 161 du STF); ce serait le cas d'une convention - valide -, faite au commencement des négociations, selon laquelle n'importe quelle des parties, à n'importe quel moment, pourrait rompre les négociations sans responsabilité par dommages-intérêts.

La conclusion que l'on peut tirer des paragraphes précédents c'est que la responsabilité extracontractuelle par dol (délit) est inamovible, car la clause de ne pas indemniser n'autorise pas la pratique de l'acte illicite, mais cette même responsabilité peut être éliminée dans le cas échéant de l'acte coupable (quasi-délit).

\section{Derniéres considérations}

Synthèse: Le principe de la bonne foi dans la formation des contrats, soit dans son aspect subjectif soit dans son aspect objectif de règle de comportement, a une grande application dans la vie pratique du peuple brésilien; une vérification sociologique constaterait facilement que les expressions bonne foi et mauvaise foi sont les plus utilisées quand on commente une affaire accomplie. Dans le champs juridique, néanmoins, le principe de la bonne foi objective n'est pas exprimé en règle générique sur la formation contractuelle (pas même sur l'éxécution contractuelle). Des nouvelles lois, spécialement la Constitution de la République (1988) et le Code de Protection au Consommateur (1990) révèlent, toutefois, une augmentation du numéro des dispositions qui se refèrent explicitement à la bonne foi (objective).

Première conclusion: Il y a, comme l'on sait, une certaine distance dans l'ordre d'abstraction intelectuelle entre un principe juridique, qui est une idée ordinatrice d'un ensemble de règles, et les règles juridiques dans lesquelles il se reflète; c'est une distance semblable à celle qui existe entre la règle juridique et le cas concret. L'esprit monte en deux étapes, établies sur trois plans, du cas à la règle et de la règle au principe; ce que l'on gagne en découverte, l'on perd en concrétitude. Inversement, le principe étant acquis, l'applicateur du droit descend du principe à la règle et de la règle au cas. Or, avec le principe de la bonne foi l'on ne peut pas agir différement; il s'en suit que, pour le meilleur dévéloppement de ce principe dans la formation contractuelle, il est indispensable son dédoublement en règles ainsi comme il arrive, par exemple, dans la détermination du devoir 
d'informer, de ne pas révéler ce que l'on a su dans la phase préliminaire, d'indemniser les dommages causés par la rupture des négociations. Nous croyons que cette sorte de développement est aujourd'hui en pleine occurrence dans le droit brésilien. Toutefois, à mesure que les règles sont formulées, les praticiens du droit n'ont plus besoin d'appeler pour le principe de bonne foi, qui devient ratio legis, et non, lex.

Deuxième conclusion: Dans la question spécifique de la rupture des négociations, au lieu de discuter si la responsabilité est aquilienne ou contractuelle, il serait peut-être préférable d'opter pour une troisième responsabilité, relative à la distribution équitative des pertes. La responsabilité aquilienne se base sur le principe du respect et elle a ici deux inconvénients: elle exige la preuve de la faute ce qui est difficile et elle donne droit à une réparation intégrale - qui peut être lourde. La responsabilité contractuelle, de son côté, se base sur le principe de la parole donnée et elle ne semble pas adéquate aux négociations préliminaires quand les parties n'ont pas encore effectivés l'échange de ses déclarations de volonté.

Troisième conclusion:Une règle générique de la bonne foi dans la formation contractuelle comme "notion ouverte" de laquelle le juge peut se valoir semble utile mais il y a dans cela une incongruence: la même imprécision qui facilite la décision est inconvéniente pour les parties. Comment obéir à une règle imprécise? Il semble qu'il vaudrait mieux admettre, non une règle générique dans la formation contractuelle, mais une norme suppletive qui fixerait les éxigences pour une possible indemnisation, ainsi: devant la confiance crée et les pertes souffertes, devant la rélation de cause et effet entre les négociations et les pertes (relation de causalité) et devant l'absence de la mauvaise foi du prejudiqué - trois éxigences le juge arbitrera l'indemnisation. La responsabilité pour les dommages résultants des faits dans la formation contractuelle, également à d'autres cas de responsabilité non fondée dans la faute ni dans l'inexécution des obligations, serait fondée dans le principe plus grand, de solidarité sociale, qui justifie la distribution équitative des pertes. 